\title{
Enhancing the translational capacity of $E$. coli by resolving the codon bias
}

Zoltan Lipinszki $^{\mathrm{a}, \mathrm{b}}$, Viktor Vernyik ${ }^{\mathrm{a}}$, Nora Farago ${ }^{\mathrm{c}}$, Tobias Sari ${ }^{\mathrm{a}}$, Laszlo G. Puskas ${ }^{\mathrm{c}}$, Frederick R. Blattner ${ }^{\mathrm{d}}$, Gyorgy Posfai ${ }^{\mathrm{a}, \#}$ and Zsuzsanna Gyorfy ${ }^{\mathrm{a}, \#}$

${ }^{\mathrm{a}}$ Institute of Biochemistry, Biological Research Centre of the Hungarian Academy of Sciences, Szeged, Hungary

${ }^{\mathrm{b}}$ MTA SZBK Lendület Laboratory of Cell Cycle Regulation, Biological Research Centre of the Hungarian Academy of Sciences, Szeged, Hungary

${ }^{\mathrm{c}}$ Institute of Genetics, Biological Research Centre of the Hungarian Academy of Sciences, Szeged, Hungary

${ }^{\mathrm{d}}$ Scarab Genomics LLC, Madison, WI, USA

\#corresponding authors: Zsuzsanna Gyorfy, gyorfyzs@brc.hu; Gyorgy Posfai, posfai.gyorgy@,brc.mta.hu. 


\begin{abstract}
Escherichia coli is a well-established, and popular host for heterologous expression of proteins. The preference in the choice of synonymous codons (codon bias), however, might differ for the host and the original source of the recombinant protein, constituting a potential bottleneck in production. Codon choice affects the efficiency of translation by a complex and poorly understood mechanism. The availability of certain tRNA species is one of the factors that may curtail the capacity of translation. Here we provide a tRNA-overexpressing strategy that allows the resolution of the codon bias, and boosts the translational capacity of the popular host BL21(DE3) when rare codons are encountered. In BL21(DE3)-derived strain, called SixPack, copies of the genes corresponding to the six least abundant tRNA species have been assembled in a synthetic fragment and inserted into a ribosomal RNA operon. This arrangement, while not interfering with the growth properties of the new strain, allows dynamic control of the transcription of the extra tRNA genes, providing significantly elevated levels of the rare tRNAs in exponential growth phase. Results from expression assays of a panel of recombinant proteins of diverse origin and codon composition showed that the performance of SixPack surpassed that of the parental BL21(DE3) or a related strain equipped with a rare tRNA-expressing plasmid.
\end{abstract}

Keywords: rare tRNA genes, genome editing, efficient translation, recombinant protein production, E. coli

E. coli is by far the most widely used host organism for biopharmaceutical heterologous production of recombinant proteins. This expression platform is favored for its simplicity, speed, and low cost. The codon bias discrepancy, however, can seriously hinder protein expression in $E$. 
coli $^{1-5}$. Choice in the usage of synonymous codons can be different in various organisms, and this bias has been shown to correlate with the relative and absolute quantities of individual tRNAs ${ }^{6,7}$. Heterologous expression of a protein with a high ratio of codons occurring infrequently in E. coli might deplete the corresponding tRNA species, leading to translational frameshifting, codon skipping, misincorporations, and protein truncations ${ }^{8}$. Ultimately, the codon bias seriously limits the use of E. coli as an expression platform.

To overcome this problem, codon optimization or rare tRNA overexpression strategies have been applied, with limited success. On one hand, synthesis of the recombinant protein encoding gene with an E. coli codon preference is labor-intensive and expensive. Moreover, replacing rare codons with frequent ones does not necessarily lead to increased yield in protein synthesis. Codon choice might affect expression, solubility, and folding of a protein ${ }^{9-15}$, and rare codons can, paradoxically, enhance the translation of a gene via reducing the mRNA secondary structure ${ }^{7,16,17}$. Additionally, codon optimization by gene synthesis is not feasible when testing gene libraries. On the other hand, attempts to overexpress rare tRNA species by cloning extra copies of the corresponding tRNA encoding genes into a plasmid have their drawbacks as well. In such commercially available hosts, maintenance of the tRNA-expressing plasmid requires the addition of extra antibiotics (usually the protein synthesis inhibitor chloramphenicol); moreover, the recombinant protein-encoding expression plasmid must belong to a different complementation group. Using two antibiotics and/or permanently altering the balance of the various tRNA species can have a fitness cost on the host, eventually resulting in a low success rate in applications ${ }^{10,18}$.

We sought to resolve the codon bias problem by expressing rare tRNAs in a more dynamic fashion. We hypothesized that inserting extra copies of the relevant tRNA genes into a ribosomal 
RNA ( $r r n)$ operon would harmonize their expression with the translational activity and would provide enhanced levels of rare tRNAs according to the actual needs. $r r n$ operons have key roles in bacterial physiology and economy. Synthesis of rRNA quickly reacts to environmental conditions, determining ribosome availability via regulating the expression of ribosomal proteins by a translational feedback mechanism ${ }^{19}$. In E. coli, there are seven nearly identical copies of rRNA operons. Synthesis of rRNA is driven by the strongest promoters found in the genome ${ }^{20}$, and the rate of transcription can change more than an order of magnitude between poor and rich nutrient conditions ${ }^{21}$. While most of the tRNA genes are scattered around the chromosome, all rRNA operons carry certain (but not any of the rare) tRNA genes as well, co-transcribed with the rRNA genes.

We inserted extra copies of the six tRNA genes $(\arg X, g l y T, \operatorname{leu} W, \operatorname{proL}, \arg U$, and ileX) corresponding to the minor codons of E. coli (CGG, GGA, CUA, CCC, AGA/AGG, and AUA, respectively) into one of the ribosomal RNA operons ( $r r n D)$ of BL21(DE3), the widely used $E$. coli expression host. We demonstrated that expression of these tRNA genes shows a marked increase in exponential growth phase, compared to the unmodified host. By testing the expression of a panel of recombinant proteins with a high ratio of rare codons in their genes, we showed that, in most cases, the modified strain that we named SixPack performs better and shows significantly enhanced expression of the heterologous proteins in classical IPTG-induction as well as in an auto-induction system. Moreover, compared to the commercially available strain Rosetta2(DE3)pLysS (Merck), which carries a similar array of extra tRNA genes on a plasmid (pLysSRARE2; Merck), SixPack proves to be a superior expression platform.

\section{Results}




\section{Design and genomic insertion of extra copies of tRNA genes}

Hypothetically, cloning extra copies of the genes corresponding to rare tRNAs into a rrn operon allows their controlled expression, as the activity of the operon is tightly regulated by nutrient availability and other physiological conditions ${ }^{22}$. This might ensure that expression of the rare tRNAs is coupled to the actual need for translational capacity.

The genes encoding for the six least abundant tRNA species were combined in a single, synthetic DNA fragment. The original copies of these genes are either single genes whose expression is controlled by their own promoter ( $p r o L, \arg U$, or ileX), or are parts of polycistronic operons $(\arg X, g l y T$, or $l e u W)$ (Figure S1). Since the process of maturation of the primary transcripts of tRNA genes is not thoroughly understood, the nucleotide sequences surrounding the genes in the synthetic fragment were designed to mimic the natural tRNA operons. The intergenic and flanking regions of the $\arg X$-glyT-leu $W$-proL segment were essentially identical to those of the natural $\arg X$-hisR-leuT-proM polycistronic operon. For $\arg U$ and $i l e X$, exact copies of the genes and their flanking sequences were fused to the 3' end of the segment (Figure S2).

For the genomic insertion site $r r n D$, one of the seven $r r n$ operons was selected. Although the structure, sequence, and activity of the $r r n$ operons of E. coli are very similar, the 3' end of $r r n D$ (containing $\operatorname{trh} V$ and $r r f F$ ) is unique, allowing specific targeting of the locus ${ }^{20}$.

The 1207-base synthetic operon carrying the six tRNA genes was engineered into the 3' region of $r r n D$ (Figure 1) by a multistep process using $\lambda$ RED recombineering, followed by elimination of 


\begin{abstract}
the markers via CRISPR-Cas9-stimulated homologous recombination (Figure S3), resulting in strain SixPack.
\end{abstract}

\title{
Growth properties of SixPack and control strains
}

\begin{abstract}
Altering the expression level of rare tRNAs might affect the speed of translation, co-translational folding of proteins and, consequently, the growth rate of $E$. coli ${ }^{23}$. Apparently, insertion of the extra tRNA genes into $r r n D$ did not disturb the homeostasis of the cell. The growth parameters of SixPack were identical to those of the parental BL21(DE3) in both LB and AIM (auto-induction medium). Signaling normal cellular operation, expression levels of two chaperons (DnaK and GroEL, the increase of which might be indicative of abnormal folding of native proteins) were not significantly altered (Figure S4). Noticeably, the commercially available Rosetta2(DE3)pLysS strain had a longer lag phase, lower growth rate, and lower maximum OD in both media, presumably owing to the burden of maintaining the plasmid and the presence of the antibiotic chloramphenicol in the media (Figure 2 and Table S1).
\end{abstract}

\section{Elevated expression of rare tRNAs}

Expression of the six rare tRNA genes was measured by qRT-PCR on first-strand cDNA generated from total RNA isolated from cultures of BL21(DE3) and SixPack in the exponential phase. Primers were designed to amplify both the unprocessed and the matured forms of the various tRNA species. In the case of SixPack, the measured rare tRNAs represented transcripts originating both from their original genomic copies and from the newly inserted fragment in $r r n D$. The rare tRNA ratios were normalized with the ratios of three control (abundant) tRNA species. 


\author{
Compared to parental BL21(DE3), SixPack showed elevated expression (1.8- to 3.8-fold) of the \\ six rare tRNAs (Figure 3).
}

\begin{abstract}
Despite being on the same cistron and transcribed together, increases in the expression levels of the extra tRNA genes were diverse ( $\arg U$ showing the highest and $g l y T$ the lowest increase). This might be due to several factors, including tRNA processing, stability, regulation, and diverse expression levels of the original genomic copies.
\end{abstract}

\title{
Individual impact of elevated levels of the rare tRNAs on protein expression
}

The functional effect of the increased levels of rare tRNAs was first tested separately for each tRNA species. To magnify the effect of rare tRNAs, we constructed IPTG-inducible, pETDuet-1based (Novagen) test plasmids expressing modified versions of the GFP gene under the regulation of the $\mathrm{T} 7$ promoter. GFP genes carrying runs of rare codons corresponded to each of the six extra tRNA genes (one GFP version corresponding to $\operatorname{proL}, i l e X, \arg X, g l y T$, and $\operatorname{leu} W$ each, and two GFP versions for $\arg U$, as it relates to both AGA and AGG codons). The tandem rare codons ( 3 or 5 copies) were inserted into the GFP gene right after the ATG codon. Expressions of the modified GFPs were detected by fluorescence measurements and visualized by denaturing polyacrylamide gel electrophoresis (SDS-PAGE).

\footnotetext{
IPTG- (or lactose-) induced expression of the GFP and its variants with rare codons had a negative but diverse impact on the growth of the strains. In LB medium, SixPack and BL21(DE3) usually showed similar growth patterns, and in most cases displayed higher growth parameters than Rosetta2(DE3)pLysS (Figure S5). In AIM, peculiarly, Rosetta2(DE3)pLysS reached the highest maximal optical density when GFP variants with rare codons were introduced (Figure
} 
S6). The reason might lie in the low metabolic burden, attributable to the inefficient expression of the particular protein. (We note that using standard AIM, the induction of the T7 polymerase and, consequently, T7 promoter-driven genes might be below full capacity in Rosetta2(DE3)pLysS, and this might contribute to the low expression level.)

Total fluorescence measurements of GFP production, however, showed a clear advantage of SixPack in both LB (Figure 4) and AIM (Figure S7). In both media, SixPack showed higher expression of the modified GFPs than the parental BL21(DE3) did. In one exception, there was no difference (3xGGA-GFP; GGA codon relates to glyT) (Figure S7H). Compared to Rosetta2(DE3)pLysS, SixPack showed higher expression in all cases. As an additional control, expression of GFP and 5xAGA-GFP were also tested in the other commercial strain Rosetta2(DE3). This strain, presumably due to the lack of T7 lysozyme, performed better than Rosetta2(DE3)pLysS (where introduction of LysS is intended to decrease background expression of the cloned gene), however, the expression levels were significantly lower than in SixPack (Figure S8).

Results of the fluorescence measurements were further supported by protein gel assays. Equal amounts of proteins obtained from crude extracts of cultures collected after a $10 \mathrm{~h}$ incubation were analyzed on Coomassie Brilliant Blue-stained SDS-PAGE gels. Differences in the amounts of modified GFPs in the three strains were in clear correlation with the fluorescence data (Figure S9).

\section{Heterologous protein expression performance of SixPack and control strains}




\begin{abstract}
The usefulness of SixPack was further tested by expressing a panel of eight protein-coding genes of diverse length and rare codon content (Table S2), selected from various species, including Drosophila melanogaster, Pyrococcus furiosus, Saccharomyces cerevisiae, Streptococcus pyogenes, and human papillomavirus (HPV). The genes were cloned into pETDuet-1 and expressed in SixPack and control strains under two different conditions (LB and AIM).
\end{abstract}

The growth curves of IPTG- (or lactose-) induced cultures seemed to be quite diverse in LB (Figure S10), but rather uniform in AIM (Figure S11). Production of the specific proteins was visualized by using Coomassie-stained gels and Western blotting (Figure 5). In most cases, SixPack proved to be the best producer. This was especially evident when the ratio of rare codons was higher than $8 \%$ (and the ratio of tandems was also high), as in the cases of HumdCas 9 (dCas9 optimized to human codon preference), HumdCas9-GFP fusion, and $P f u$ DNA polymerase. In the case of EFT1, the ratio was only $4.3 \%$ (35 AGA and 2 CTA codons); but SixPack still produced the highest amount of this protein. It is conceivable that the translation efficiency is influenced not only by the total amount of rare codons, but also by the occurrence of tandem situations, the location of rare codons in the gene (e.g., close or far from the start site), and the ratios of the different rare codon species ${ }^{3}$. In other cases (As1, dCas9, Flfl, and HPV16 L2), when the rare codon ratio was below $8 \%$, production by SixPack was similar to that of BL21(DE3) in LB and similar to or better than that in AIM. In all cases, the performance of Rosetta2(DE3)pLysS lagged behind that of SixPack (especially evidently in AIM) or was similar at most (Figure 6).

Usefulnesss of SixPack was further demonstrated by comparing the production of two versions of the same protein (dCas9, 5.4\% rare codons vs. HumdCas9, 8,6\% rare codons), which can be 
viewed as rare codon-containing and codon-optimized versions of the same protein. In this particular case, higher expression of HumdCas9 by SixPack versus dCas9 by BL21(DE3) clearly demonstrated the advantage of SixPack (Figure S12).

\section{Discussion}

Depletion of rare tRNA species evoked by the expression of heterologous proteins can compromise product yield and translational fidelity. Several attempts, both genetic (overexpression of rare tRNA genes from a plasmid, or codon optimization of the heterologous gene by synthesis) and non-genetic ones (optimizing the growth conditions, or using in vitro protein synthesis systems) have been applied with varying degrees of success ${ }^{2,24-28}$. We offer here a solution that resolves codon bias discrepancies with a high success rate, does not require additional antibiotics, and performs better or as well as the widely used BL21(DE3) E. coli strain.

The novelty of the approach lies in placing the extra copies of rare tRNA genes into a ribosomal operon of the host chromosome. This arrangement offers the following benefits: (i) lack of physiological burden associated with plasmid replication and maintenance, (ii) genetic stability without the use of an antibiotic, and (iii) dynamic changes in the expression of rare tRNA genes, paralleling the actual translational activity.

\footnotetext{
A ribosomal operon is a natural choice as a location for inserting tRNA genes. While most tRNA genes are scattered elsewhere in the genome, each of the rrn operons carries at least one tRNA gene (typically coding for abundant tRNAs). As anticipated, increased amounts of the rare tRNAs, corresponding to the inserted genes, were detected. However, when compared to the
} 
levels in the parental host BL21(DE3), the rate of increase in the exponential phase was only modest. An interplay of several factors, including co-factors of tRNA maturation, tRNA stability, and feedback regulation of the genes at the original locations, might mitigate the increase. This limited elevation in rare tRNA abundance might even be a fortuitous feature. Apparently, the increase is not high enough (the rare tRNAs remain minor components in the tRNA pool) to disturb normal physiological operation.

The modest overexpression of the rare tRNAs had, however, a significant, positive effect on heterologous protein expression, mitigating the effect of tRNA depletion. Individually, with the exception of $g l y T$ in AIM, all the genes caused increased expression (up to 12-fold) of specific GFP variants carrying tandem copies of the corresponding rare codon. The combined effect of the six extra genes was then clearly demonstrated by expressing a panel of proteins of various origin and codon bias. Expression in SixPack (amount of target protein per cell mass or per culture volume) was at least as good as in any of the control strains and in fact, was better in most cases (in some cases even 20-fold). In general, with the increasing number of rare codons and their tandem copies, the advantage of SixPack became increasingly evident. SixPack compared especially favorably to control strains when applying AIM, which is a popular medium for biotechnological production of proteins in large volumes (i.e., in bioreactors) ${ }^{29}$.

\footnotetext{
The aim of this study was to improve the performance of BL21(DE3) for the heterologous expression of proteins. We do not claim that other strategies, like codon optimization, cannot work better in certain cases, but the simplicity of the use of SixPack is definitely an attractive feature.
} 


\begin{abstract}
In summary, insertion of the six rare tRNA genes into the genome of BL21(DE3) did not result in changes in its basic characteristics like morphology, growth properties, or transformation efficiency. The beneficial features (no need for extra plasmid and antibiotics, no need for codon optimization, simplicity when expressing gene libraries, high success rate when expressing proteins with different codon bias) render SixPack a useful, and likely superior, alternative host for expression of heterologous proteins.
\end{abstract}

\title{
Materials and Methods
}

\section{Medium}

In all experiments involving bacterial culture growth, standard LB or AIM (auto-induction medium; LB broth base including trace elements; Formedium LTD, England) were used. Antibiotics were used in the following concentrations: $100 \mu \mathrm{g} / \mathrm{ml}$ ampicillin (Ap) and $24 \mu \mathrm{g} / \mathrm{ml}$ chloramphenicol (Cam).

\section{E. coli strains}

BL21(DE3) was used as the parental strain. It expresses T7 RNA polymerase from the $\lambda$ DE3 lysogen inserted into the genome under the regulation of the lacUV5 promoter. This polymerase can drive the expression of the genes of interest via the T7 promoter $^{30}$.

Rosetta2(DE3)pLysS is a derivative of BL21(DE3). In this strain, pLysSRARE2 plasmid encodes seven genes of rare tRNAs $(\arg U, \arg X, \arg W, g l y T, l e u W$, ileX, and proL, recognizing the AGA/AGG, CGG, AGG, GGA, CUA, AUA, and CCC codons, respectively) under the control of 
their own promoter. pLysSRARE2 also contains chloramphenicol resistance- and lysosymeencoding genes ${ }^{31}$.

To create the SixPack strain, BL21(DE3) was modified by inserting extra copies of six rare tRNA genes $(\arg U, \arg X, g l y T, \operatorname{leu} W$, ileX, and $\mathrm{proL})$ into its $r r n D$ operon (Figure 1). These tRNAs recognize the same rare codons as the tRNAs expressed from the pLysSRARE2 plasmid (AGG is recognized by $\arg X$ and $\arg W$ in Rosetta2(DE3)pLysS, but only by $\arg X$ in SixPack). Rosetta2(DE3) strain (Merck) supplies the same tRNA genes on pRARE2 plasmid, without lysozyme expression.

\section{Genomic construct}

The rare tRNA genes were cloned into pSG76A (replicating by R6K ori) in three pieces. The first part (synthesized by Thermo Fisher Scientific GENEART GmbH; Germany) contained the $\arg X$, glyT, leuW, and proL tRNA genes. The structure of this DNA fragment was based on the polycistronic operon of $E$. coli coding for $\arg X$, hisR, leuT, and proM tRNA genes; we replaced the hisR, leuT, and proM genes with glyT, leuW, and proL rare tRNA genes, but kept argX and the original intergenic regions. The second and third pieces coding for $\arg U$ and ileX, respectively, were directly amplified from the $E$. coli genome with their own intergenic regions (Figures S1 and S2). The plasmid carrying the six tRNA genes was linearized by PCR using overhanging primers containing homologous regions (50- or 100-nt) with the target site (unique sequence of the 3' end of $r r n D$ ), and transformed into BL21(DE3) in the presence of $\lambda$ RED recombinase ${ }^{32}$. After successful recombination into the genome, the CRISPR-Cas9 system was used to introduce a double-strand break in the ampicillin resistance gene on the insert ${ }^{33}$. The cells' own RecA system repaired the cleavage by homologous recombination using the 47-nt box 
on the plasmid homologous to the adjacent $r r n D$ sequence downstream from the site of insertion (Figures S3 and S13) ${ }^{34}$.

\section{Expression plasmids}

The coding sequences of different test proteins were cloned into pETDuet-1 (Novagen). A list of these genes and the cloning sites is given in Table S3. The GFP-encoding gene was amplified from pCA24N (the cloning plasmid of the ASKA collection) ${ }^{35}$, and subcloned into pETDuet-1. GFP gene variants containing runs of rare codons were created by PCR using $\mathrm{pETDUET/GFP} \mathrm{as}$ a template. The coding DNA sequence of asterless (Asl) was subcloned from the GH02902 Drosophila Gold cDNA clone into pETDuet-1 following standard procedures. Cloning of Flf1 into pETDuet-1 has been described elsewhere ${ }^{36}$. dCas 9 was cloned from the pdCas 9 plasmid \# 46569 from Addgene ${ }^{37}$. The HumdCas9 gene was amplified from pMLM3705 plasmid \# 47754 from Addgene ${ }^{38}$. pETDUET/HumdCas9-GFP expresses HumdCas9 and GFP as a fusion protein. EFT1, the gene of elongation factor 2, was amplified from genomic DNA of Saccharomyces cerevisiae strain EMY 74.7 and was a kind gift from Tamas Feher ${ }^{39}$.

The HPV16 L2 gene (subcloned in Addgene plasmid \# 72473) was a kind gift from Vilmos Tubak. The gene of Pfu DNA polymerase was amplified from Pyrococcus furiosus genomic DNA (DSM 3638) which was a kind gift from Vilmos Tubak. BL21(DE3) harbouring an empty pETDuet-1 was used as a control.

\section{Quantitative real-time PCR (qRT-PCR)}

RNA was isolated from exponential phase cultures grown in LB, using the E.Z.N.A. Bacterial RNA Kit (VWR, USA). To obtain cDNA, $1 \mu \mathrm{g}$ of RNA was then reverse transcribed using the High-Capacity cDNA Reverse Transcription Kit (Thermo Fisher Scientific) in a final volume of 
$10 \mu \mathrm{l}$ by using 1 pmole of the reverse primers (Table S4). After dilution with $20 \mu \mathrm{l}$ of water, $1 \mu \mathrm{l}$ of the diluted reaction mixture was used as a template in qRT-PCR with $10 \mu \mathrm{l}$ of qPCRBIO SyGreen Mix (PCR Biosystems) and $1 \mu \mathrm{l}$ of gene-specific primer mix, according to the following protocol: $10 \mathrm{~min}$ at $95^{\circ} \mathrm{C}$ followed by 40 cycles of $95^{\circ} \mathrm{C}$ for $25 \mathrm{sec}, 60^{\circ} \mathrm{C}$ for $25 \mathrm{sec}$, and $72^{\circ} \mathrm{C}$ for 15 sec. qRT-PCR was performed in a LightCycler ${ }^{\circledR}$ Nano Real-Time PCR System (Roche Diagnostics GmbH; Mannheim, Germany). Transcripts of proK, alaU, and gltW genes were used as house-keeping tRNA probes.

\section{Measuring and calculating growth parameters}

To measure growth parameters, a Synergy 2 automated microplate reader machine (BioTek, USA) was used. Aliquots of $1 \mu$ leach of BL21(DE3), SixPack, and Rosetta2(DE3)pLysS overnight starter cultures were transferred into $100 \mu \mathrm{l}$ fresh LB or AIM medium in dedicated 96well plates. Rosetta2(DE3)pLysS cultures were supplemented with Cam. Absorbance at $600 \mathrm{~nm}$ was measured every 5 min for $24 \mathrm{~h}$ at $37^{\circ} \mathrm{C}$ with continuous shaking. Growth parameters (length of the lag phase, doubling time and OD increment) were calculated by using previously described methods ${ }^{40}$.

\section{Fluorescence measurements}

Starter cultures of BL21(DE3), SixPack, Rosetta2(DE3)pLysS and Rosetta2(DE3) strains expressing GFP or GFP variants were grown overnight in LB in a Synergy 2 automated microplate reader machine (BioTek, USA). Starter cultures $(1 \mu$ leach) were re-inoculated into $100 \mu \mathrm{l}$ fresh LB or AIM media supplemented with Ap and isopropyl $\beta$-D-1-thiogalactopyranoside (IPTG) (plus Cam, in the case of Rosetta2(DE3)pLysS and Rosetta2(DE3)). IPTG was used in 


\begin{abstract}
concentrations optimized for highest induction (0.05 mM for BL21(DE3) and SixPack, $0.1 \mathrm{mM}$ for Rosetta2(DE3) and $0.5 \mathrm{mM}$ for Rosetta2(DE3)pLysS). Measurements were made every 5 $\min$.
\end{abstract}

\title{
Protein expression and whole cell lysate preparation
}

Protein expressions in BL21(DE3) and SixPack were induced with $0.05 \mathrm{mM}$ IPTG, and those in Rosetta2(DE3)pLysS were induced with $0.5 \mathrm{mM}$ IPTG in $3 \mathrm{ml}$ of LB or AIM (supplemented with antibiotics) for $10 \mathrm{~h}$. Bacteria were harvested by centrifugation, resuspended in $150 \mu \mathrm{l} /$ absorption unit (at $600 \mathrm{~nm}$ ) of phosphate-buffered saline, $\mathrm{pH} 7.4$, supplemented with $1 \mathrm{U} / \mathrm{ml}$ BenzonaseNuclease (Merck) and $2 \mathrm{mM} \mathrm{MgCl}_{2}$, kept on ice for $10 \mathrm{~min}$, mixed with 4x Laemmli sample buffer and boiled for $5 \mathrm{~min}$.

\section{SDS-PAGE and Western blotting}

Equal amounts of protein samples were run on $7 \%$ or $10 \%$ SDS-PAGE gels. Gels were fixed for $10 \mathrm{~min}$ in $10 \%$ acetic acid, stained for $15 \mathrm{~min}$ with Coomassie Brilliant Blue $(0.1 \% \mathrm{CBB}$ in $50 \%$ methanol and 10\% acetic acid), and differentiated overnight in 7\% acetic acid and $10 \%$ methanol. For immunoblotting, proteins were blotted onto a nitrocellulose membrane (GE Healthcare) and probed with anti-His mouse monoclonal (Thermo Fisher Scientific, \#MA1-21315; dilution 1:35000), anti-Flfl rat polyclonal ${ }^{36}$ (dilution 1: 15,000), anti-Asl rabbit polyclonal ${ }^{41}$ (dilution 1 : 20,000), anti-GroEL rabbit polyclonal ${ }^{42}$ (dilution 1:5,000) or anti-DnaK1 mouse monoclonal (dilution 1:5,000, Stressgen SPA-880) antibodies following standard procedures. Stained gels and X-ray films were scanned at 600 dpi resolution for image processing.

\section{Funding}


This work was supported by The National Research, Development and Innovation Office (OTKA K116455 to GP and OTKA-PD1 15404 to ZL), the Ministry for National Economy of Hungary (GINOP-2.3.2-15-2016-00001), the Hungarian Academy of Sciences (Bolyai Fellowship (bo_329_15), and Lendület Program Grant (LP2017-7/2017) to ZL).

\section{Acknowledgements}

The authors would like to thank Vilmos Tubak (BRC) for the Pirococcus furiosus genomic DNA and the HPV16 L2-expressing plasmid, Tamas Feher (BRC) for the EFT1-coding plasmid, and Laszlo Vigh (BRC) for DnaK1 and GroEL antibodies, David Glover (University of Cambridge) for Asl antibody, and the Drosophila Genomics Resource Center (NIH grant 2P40OD010949) for asterless cDNA.

\section{References}

1. Deana, A., Ehrlich, R., and Reiss, C. (1998) Silent mutations in the Escherichia coli ompA leader peptide region strongly affect transcription and translation in vivo. Nucleic Acids Res. 26, 4778-4782.

2. Novy, R., Drott, D., Yaeger, K., and Mierendorf, R. (2001) Overcoming the codon bias of E. coli for enhanced protein expression. In Innovations, Newsletter of Novagen, Inc. 12, 1-4.

3. Kim, S., and Lee, S.-B. (2006) Rare codon clusters at 5'-end influence heterologous expression of archaeal gene in Escherichia coli. Protein Expr. Purif. 50, 49-57. 
4. Harris, R.-P., and Kilby, P.-M. (2014) Amino acid misincorporation in recombinant biopharmaceutical products. Curr. Opin. Biotechnol. 30, 45-50.

5. Boël, G., Letso, R., Neely, H., Price, W.-N., Wong, K.-H., Su, M., Luff, J., Valecha, M., Everett, J.-K., Acton, T.-B., Xiao R., Montelione, G.-T., Aalberts, D.-P., and Hunt, J.-F. (2016) Codon influence on protein expression in E. coli correlates with mRNA levels. Nature 529, 358-363.

6. Ikemura, T. (1981) Correlation between the abundance of Escherichia coli transfer RNAs and the occurrence of the respective codons in its protein genes: a proposal for a synonymous codon choice that is optimal for the E. coli translational system. J. Mol. Biol. 151, 389-409.

7. Novoa, E.-M., and Ribas de Pouplana, L. (2012) Speeding with control: codon usage, tRNAs, and ribosomes. Trends Genet. 28, 574-581.

8. McNulty, D.-E., Claffee, B.-A., Huddleston, M.-J., and Kane, J.-F. (2003) Mistranslational errors associated with the rare arginine codon CGG in Escherichia coli. Protein Expr. Purif. 27, $365-374$.

9. Thangadurai, C., Suthakaran, P., Barfal, P., Anandaraj, B., Pradhan, S.-N., Boneya, H.-K., Ramalingam, S., and Murugan, V. (2008) Rare codon priority and its position specificity at the 5' of the gene modulates heterologous protein expression in Escherichia coli. Biochem. Biophys. Res. Commun. 376, 647-652.

10. Kudla, G., Murray, A.-W., Tollervey, D., and Plotkin, J.-B. (2009) Coding-sequence determinants of gene expression in Escherichia coli. Science 324, 255-258.

11. Rosano, G.-L., and Ceccarelli, E.-A. (2009) Rare codon content affects the solubility of recombinant proteins in a codon bias-adjusted Escherichia coli strain. Microb. Cell Fact. 8, 41. 12. Mauro, V.-P., and Chappell, S.-A. (2014) A critical analysis of codon optimization in human therapeutics. Trends Mol. Med. 20, 604-613. 
13. Borel, F., Härtlein, M., and Leberman, R. (1993) In vivo overexpression and purification of Escherichia coli tRNA(ser). FEBS Lett. 324, 162-166.

14. Pavon-Eternod, M., Gomes, S., Rosner, M.-R., and Pan, T. (2013) Overexpression of initiator methionine tRNA leads to global reprogramming of tRNA expression and increased proliferation in human epithelial cells. $R N A 19,461-466$.

15. Shaham, G., and Tuller, T. (2018) Genome scale analysis of Escherichia coli with a comprehensive prokaryotic sequence-based biophysical model of translation initiation and elongation. DNA Res. 25, 195-205.

16. Goodman, D.-B., Church, G.-M., and Kosuri, S. (2013) Causes and effects of N-terminal codon bias in bacterial genes. Science 342, 475-479.

17. Karimi, Z., Nezafat, N., Negahdaripour, M., Berenjian, A., Hemmati, S., and Ghasemi, Y. (2015) The effect of rare codons following the ATG start codon on expression of human granulocyte-colony stimulating factor in Escherichia coli. Protein Expr. Purif. 114, 108-114.

18. Søgaard, K.-M., and Nørholm, M.-H. (2016) Side effects of extra tRNA supplied in a typical bacterial protein production scenario. Protein Sci. 25, 2102-2108.

19. Nomura, M., Yates, J.-L., Dean, D., and Post, L.-E. (1980) Feedback regulation of ribosomal protein gene expression in Escherichia coli: structural homology of ribosomal RNA and ribosomal protein mRNA. Proc. Natl. Acad. Sci. U.S.A. 77, 7084-7088.

20. Jin, D.-J., Cagliero, C., and Zhou, Y.-N. (2012) Growth rate regulation in Escherichia coli. FEMS Microbiol. Rev. 36, 269-287.

21. Milo, R., and Phillips, R. (2015) How many ribosomes are in the cell? In Cell biology by the numbers. Garland Science. ISBN13 9780815345374

22. Paul, B.-J., Ross, W., Gaal, T., and Gourse, R.-L. (2004) rRNA transcription in Escherichia coli. Annu. Rev. Genet. 38, 749-770. 
23. Tuller, T., and Zur, H. (2015) Multiple roles of the coding sequence 5' end in gene expression regulation. Nucleic Acids Res. 43, 13-28.

24. Mualif, S.-A., Teow, S.-Y., Omar, T.-C., Chew, Y.-W., Yusoff, N.-M., and Ali, S.-A. (2015) Engineering and validation of a vector for concomitant expression of rare transfer RNA (tRNA) and HIV-1 nef Genes in Escherichia coli. PloS One 10:e130446.

25. Burgess-Brown, N.-A., Sharma, S., Sobott, F., Loenarz, C., Oppermann, U., and Gileadi, O. (2008) Codon optimization can improve expression of human genes in Escherichia coli: A multi-gene study. Protein Expr. Purif. 59, 94-102.

26. Hutterer, K.-M., Zhang, Z., Michaels, M.-L., Belouski, E., Hong, R.-W., Shah, B., Berge, M., Barkhordarian, H., Le, E., Smith, S., Winters, D., Abroson, F., Hecht, R., and Liu, J. (2012) Targeted codon optimization improves translational fidelity for an Fc fusion protein. Biotechnol. Bioeng. 109, 2770-2777.

27. Chen, H., Xu, Z., and Cen, P. (2006) High-level expression of human beta-defensin-2 gene with rare codons in E. coli cell-free system. Protein Pept. Lett. 13, 155-162.

28. Chumpolkulwong, N., Sakamoto, K., Hayashi, A., Iraha, F., Shinya, N., Matsuda, N., Kiga, D., Urushibata, A., Shirouzu, M., Oki, K., Kigawa, T., and Yokoyama, S. (2006) Translation of 'rare' codons in a cell-free protein synthesis system from Escherichia coli. J. Struct. Funct.

Genomics 7, 31-36.

29. Studier, F.-W. (2005) Protein production by auto-induction in high-density shaking cultures. Protein Expr. Purif. 41, 207-234.

30. Studier, F.-W., Rosenberg, A.-H., Dunn, J.-J., and Dubendorff, J.-W. (1990) Use of T7 RNA polymerase to direct expression of cloned genes. Methods Enzymol. 185, 60-89.

31. Studier, F.-W. (1991) Use of bacteriophage T7 lysozyme to improve an inducible T7 expression system. J. Mol. Biol. 219, 37-44. 
32. Muyrers, J.-P., Zhang, Y., Testa, G., and Stewart, A.-F. (1999) Rapid modification of bacterial artificial chromosomes by ET-recombination. Nucleic Acids Res. 27, 1555-1557.

33. Jiang, W., Bikard, D., Cox, D., Zhang, F., and Marraffini, L.-A. (2013) RNA-guided editing of bacterial genomes using CRISPR-Cas systems. Nat. Biotechnol. 31, 233-9.

34. Kowalczykowski, S.-C. (2000) Initiation of genetic recombination and recombinationdependent replication. Trends Biochem. Sci. 25, 156-165.

35. Kitagawa, M., Ara, T., Arifuzzaman, M., Ioka-Nakamichi, T., Inamoto, E., Toyonaga, H., and Mori, H. (2005) Complete set of ORF clones of Escherichia coli ASKA library (a complete set of E. coli K-12 ORF archive): unique resources for biological research. DNA Res. 12, 291-299.

36. Lipinszki, Z., Lefevre, S., Savoian, M.-S., Singleton, M.-R., Glover, D.-M., and Przewloka, M.-R. (2015) Centromeric binding and activity of Protein Phosphatase 4. Nat. Commun. 6, 5894.

37. Bikard, D., Jiang, W., Samai, P., Hochschild, A., Zhang, F., and Marraffini, L.-A. (2013) Programmable repression and activation of bacterial gene expression using an engineered CRISPR-Cas system. Nucleic Acids Res. 41, 7429-7437.

38. Maeder, M.-L., Linder, S.-J., Cascio, V.-M., Fu, Y., Ho, Q.-H., and Joung, J.-K. (2013) CRISPR RNA-guided activation of endogenous human genes. Nat. Methods 10, 977-979. 39. Johnson, R.-E., Torres-Ramos, C.-A., Izumi, T., Mitra, S., Prakash, S., and Prakash, L. (1998) Identification of APN2, the Saccharomyces cerevisiae homolog of the major human AP endonuclease $H A P 1$, and its role in the repair of abasic sites. Genes Dev. 12, 3137-3143.

40. Warringer, J., Ericson, E., Fernandez, L., Nerman, O., and Blomberg, A. (2003) Highresolution yeast phenomics resolves different physiological features in the saline response. Proc. Natl. Acad. Sci. U.S.A. 100, 15724-15729. 
41. Dzhindzhev, N.-S., Yu, Q.-D., Weiskopf, K., Tzolovsky, G., Cunha-Ferreira, I., Riparbelli, M., Rodrigues-Martins, A., Bettencourt-Dias, M., Callaini, G., and Glover, D.-M. (2010)

Asterless is a scaffold for the onset of centriole assembly. Nature 467, 714-718.

42. Lehel, C., Wada, H., Kovacs, E., Torok, Z., Gombos, Z., Horvath, I., Murata, N., and Vigh, L. (1992) Heat shock protein synthesis of the cyanobacterium Synechocystis PCC 6803:

purification of the GroEL-related chaperonin. Plant Mol. Biol. 18, 327-36.

\section{Figure legends}

Figure 1. Schematic picture of $r r n D$ showing the six extra tRNA genes (dark blue) inserted in the 3' end region between thrV and $r r f F$.

Figure 2. Growth curves of SixPack and control strains BL21(DE3) (marked BL) and Rosetta2(DE3)pLysS (marked R) in LB (A) and in AIM (B). (Averages of three independent experiments.) There is no significant difference between the growth parameters of BL21(DE3) and SixPack (two sample t-test) (see also Table S2).

Figure 3. Ratios of the different tRNA species between SixPack and BL21(DE3) in the log phase. (Averages of five independent experiments, each comprising three technical repetitions.) Statistical analyses were performed on ddCt values (one-sample t-test, against zero); $\mathrm{p}<0,05$ was measured in all cases.

Figure 4. Expression of GFP (A) and modified GFP versions (B-H) in BL21(DE3) (marked BL), SixPack, and Rosetta2(DE3)pLysS (marked R) in LB, monitored by fluorescence measurements. 


\begin{abstract}
(The curves represent the averages of four independent experiments.) Statistical analysis was performed on 20 h-time point fluorescence values, the differences between SixPack vs. BL21(DE3) were significant in cases $\mathbf{B}-\mathbf{F}(\mathrm{p}<0,05$; paired t-test).
\end{abstract}

Figure 5. Production of eight heterologous proteins (A-H for Asl, dCas9, HumdCas9, HumdCas9-GFP fusion, EFT1, Flfl, HPV16 L2, and Pfu, respectively) detected on protein gels by Coomassie Brilliant Blue staining and Western blotting. Proteins were extracted from BL21(DE3) (marked BL), SixPack and Rosetta2(DE3)pLysS (marked R) after $10 \mathrm{~h}$ growth in LB or AIM. Asterisks indicate cases where SixPack produced significantly higher amount of test proteins compared to BL21(DE3), based on densitometry data of Western blots of three independent experiments ( $\mathrm{p}<0,05$; paired t-test). "Empty" indicates BL21(DE3) harboring pETDuet-1.

Figure 6. Summary of heterologous protein production of BL21(DE3) (marked BL), SixPack, and Rosetta2(DE3)pLysS (marked R) measured in LB and AIM. The "+" and "-“" marks indicate the protein production of the strains relative to SixPack: 100-68\% $(+++), 67-35 \%(++), 34-2 \%(+)$, $1 \%$ or lower (-). Table $\mathbf{A}$ is based on the fluorescence values of GFP and GFP variants detected $20 \mathrm{~h}$ after induction. Table $\mathbf{B}$ is based on results of Western blots, validated by densitometry. Circles label the cases when SixPack performed better than the parental strain BL21(DE3) in a statistically significant manner. 


\section{Figures}

Figure 1.

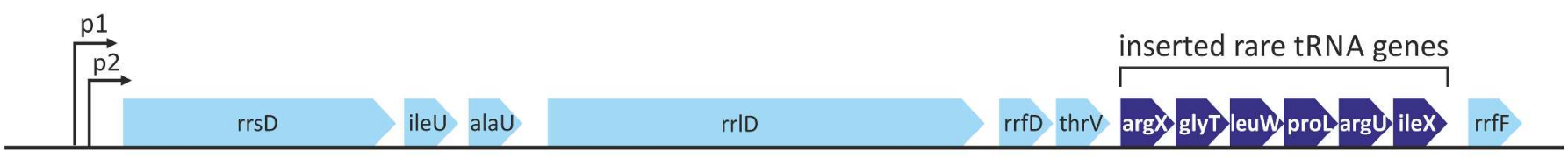

Figure 2.

A

B
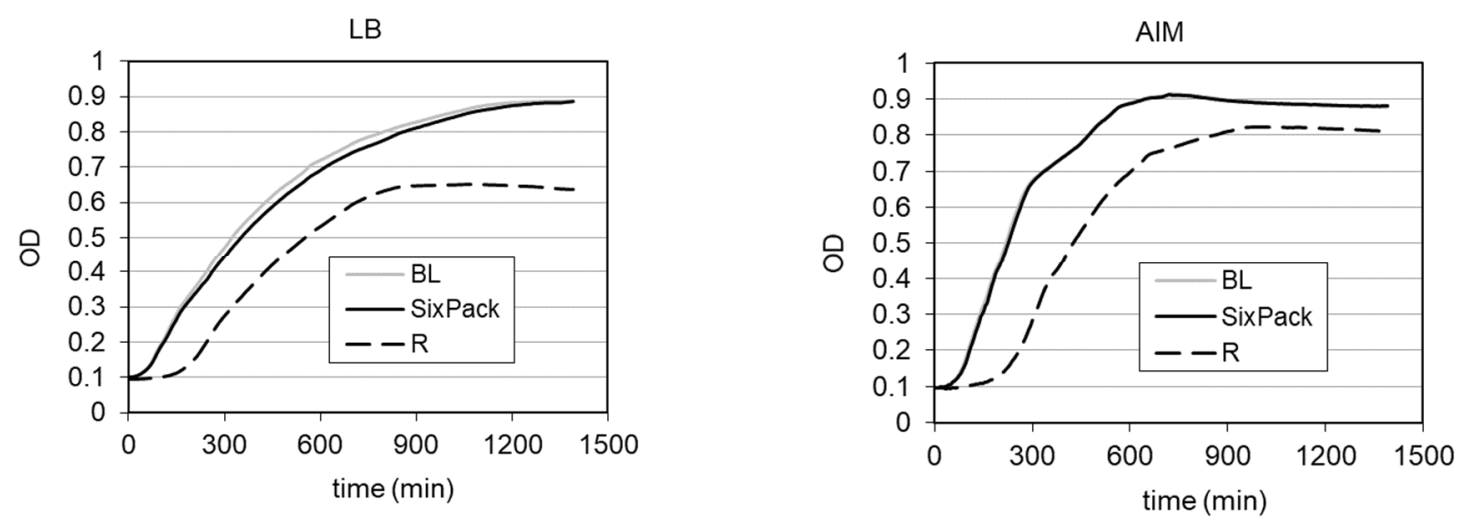
Figure 3.

fold change: SixPack vs. BL21(DE3)

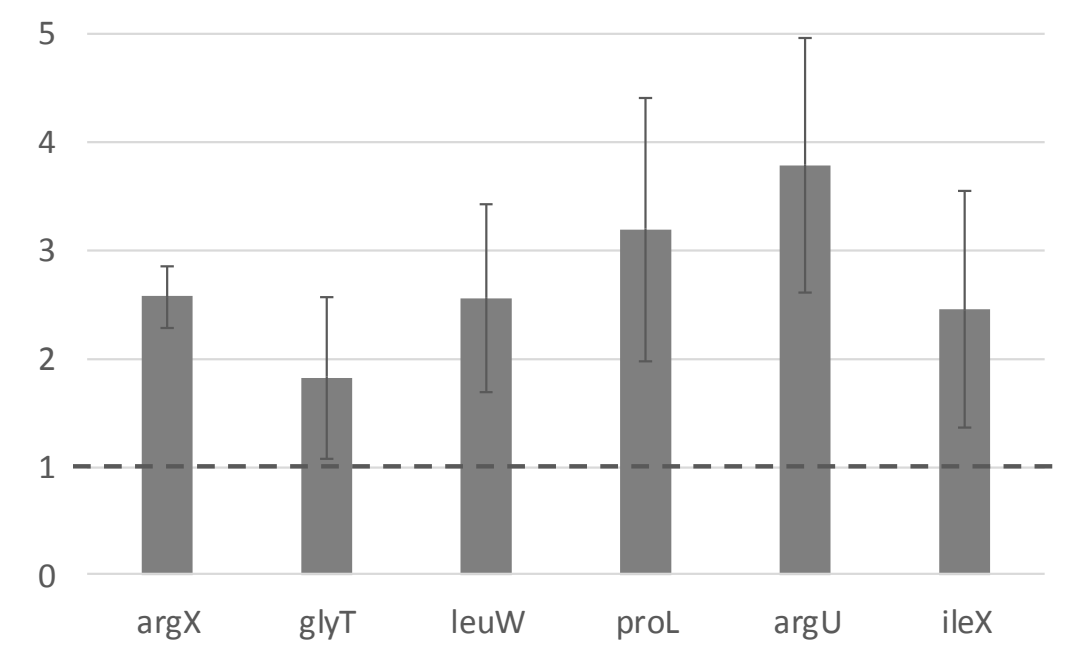


Figure 4.

A

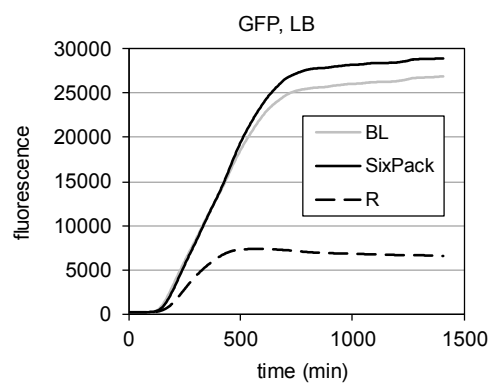

D

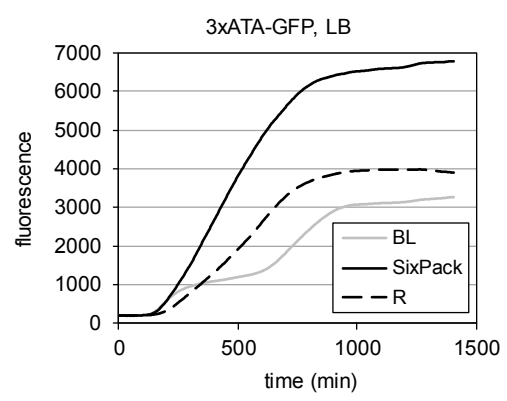

G

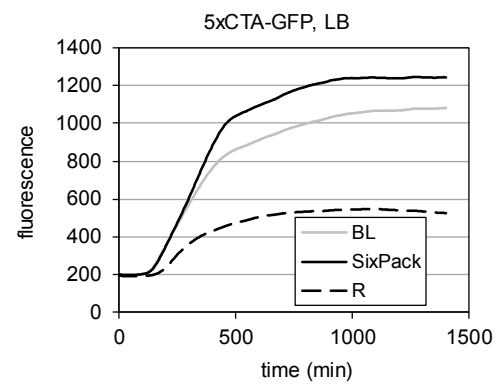

$\mathrm{H}$

B

C

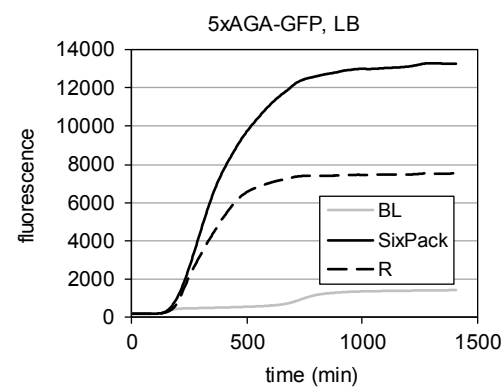

E
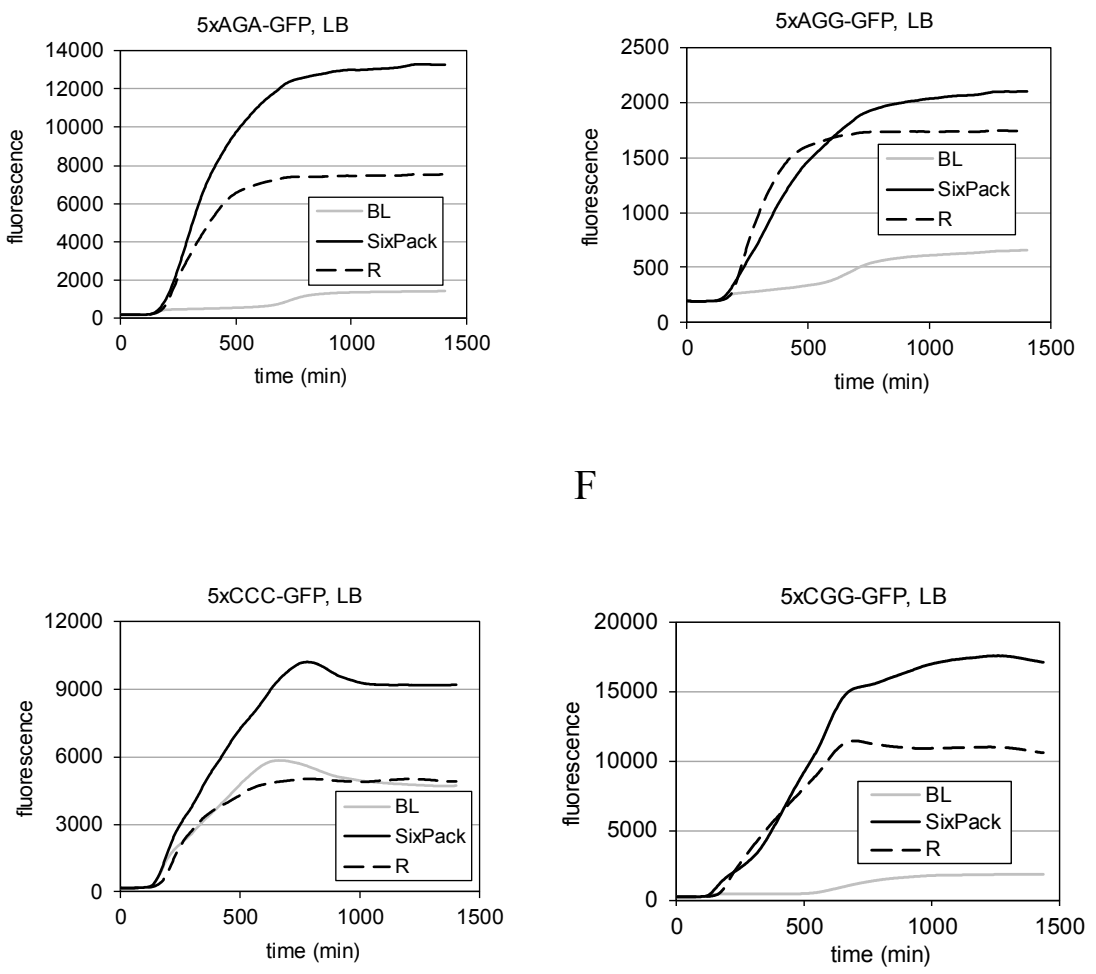

F

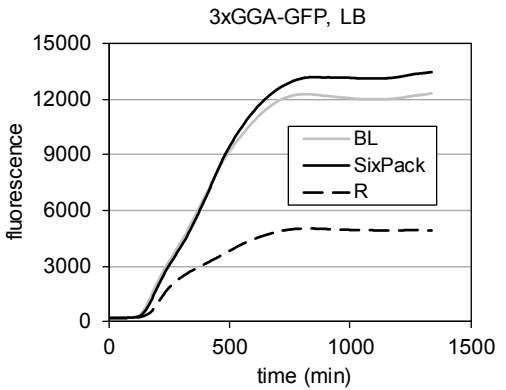


Figure 5.

A

B
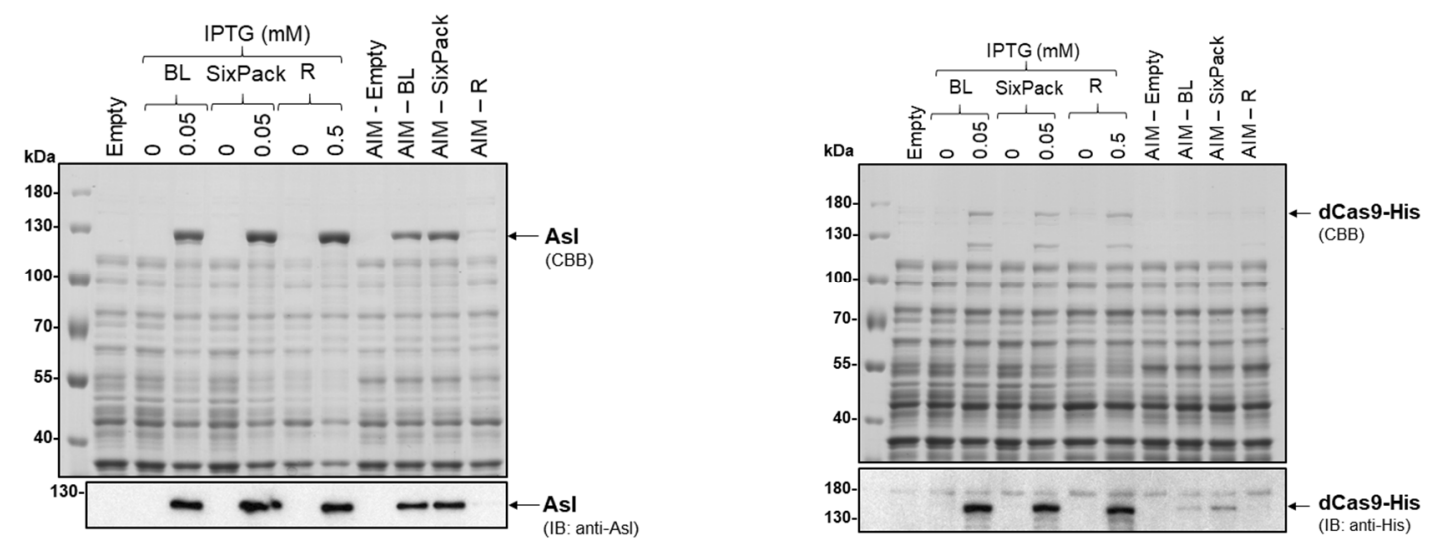

C

D
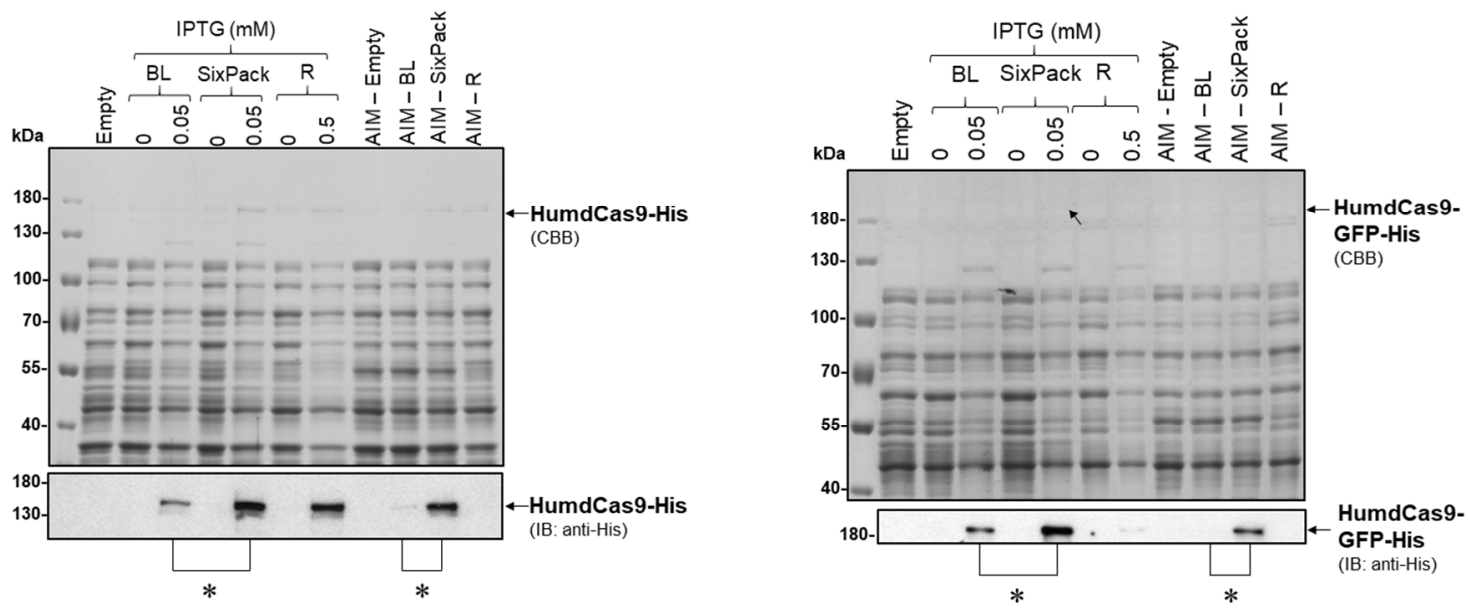

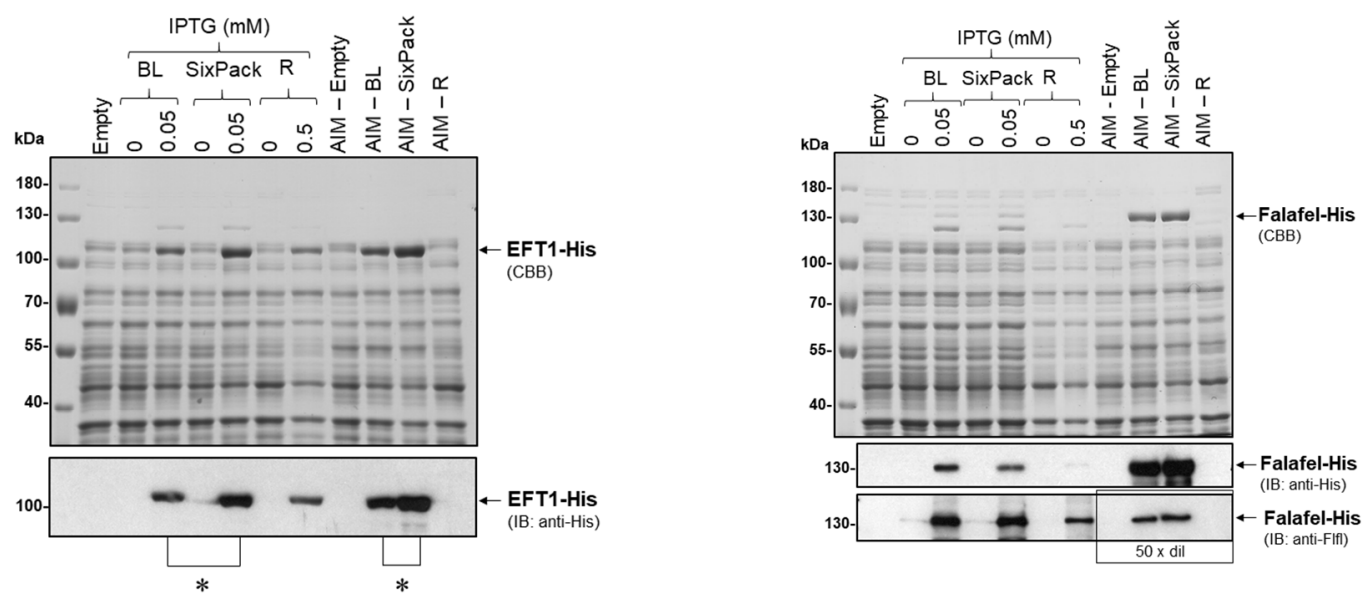

G

$\mathrm{H}$
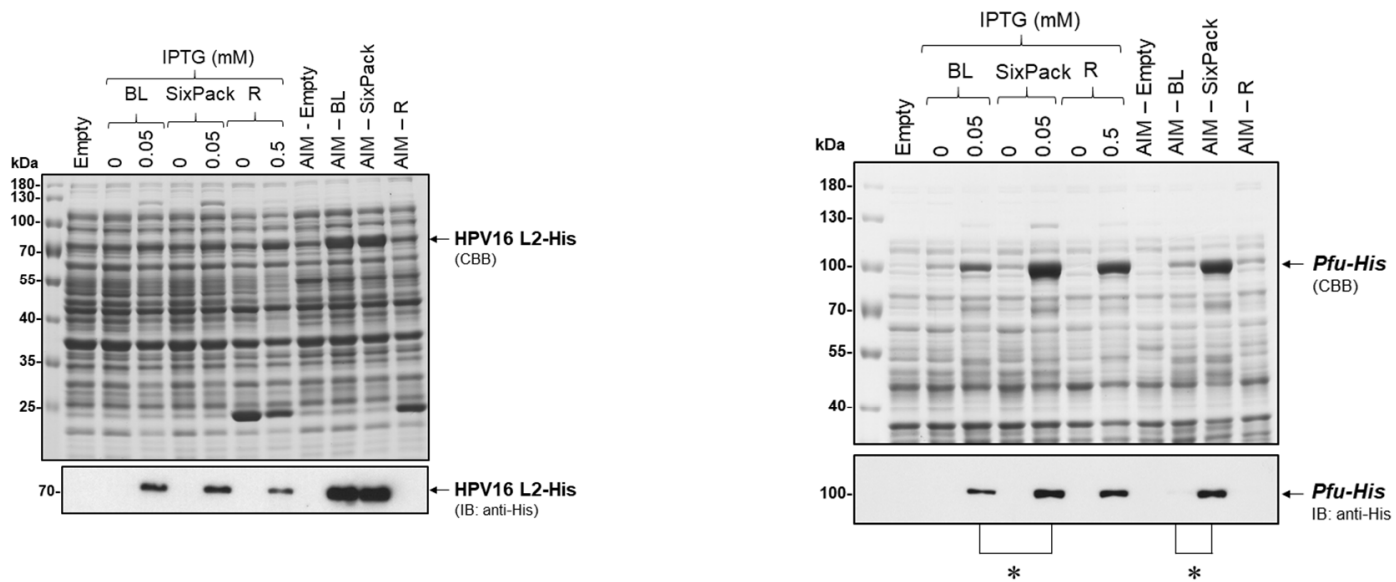
Figure 6.

A

LB, IPTG

\begin{tabular}{|c|c|c|}
\hline$B L$ & SixPack & $R$ \\
\hline+++ & +++ & + \\
\hline+ & +++ & ++ \\
\hline+ & +++ & +++ \\
\hline++ & +++ & ++ \\
\hline++ & +++ & ++ \\
\hline+ & +++ & ++ \\
\hline+++ & +++ & ++ \\
\hline+++ & +++ & ++ \\
\hline
\end{tabular}

AIM

\begin{tabular}{|c|c|c|}
\hline BL & SixPack & R \\
\hline+++ & +++ & + \\
\hline+ & +++ & + \\
\hline++ & +++ & +++ \\
\hline++ & +++ & ++ \\
\hline++ & +++ & + \\
\hline+ & +++ & ++ \\
\hline+++ & +++ & ++ \\
\hline+++ & +++ & + \\
\hline
\end{tabular}

B

LB, IPTG

\begin{tabular}{|c|c|c|}
\hline BL & SixPack & $\mathbf{R}$ \\
\hline+++ & +++ & +++ \\
\hline++ & ++ & ++ \\
\hline+ & +++ & ++ \\
\hline+ & +++ & - \\
\hline++ & +++ & + \\
\hline++ & ++ & + \\
\hline++ & ++ & + \\
\hline+ & +++ & ++ \\
\hline
\end{tabular}

$+$
AIM

\begin{tabular}{|c|c|c|}
\hline BL & SixPack & $\mathbf{R}$ \\
\hline++ & ++ & - \\
\hline+ & ++ & - \\
\hline+ & +++ & - \\
\hline- & ++ & - \\
\hline++ & +++ & - \\
\hline+++ & +++ & - \\
\hline+++ & +++ & - \\
\hline- & +++ & - \\
\hline
\end{tabular}

\begin{tabular}{|l|}
\hline Asl \\
\hline dCas9 \\
\hline HumdCas9 \\
\hline HumdCas9-GFP \\
\hline EFT1 \\
\hline Flfl \\
\hline HPV16 L2 \\
\hline Pfu \\
\hline
\end{tabular}

\begin{tabular}{|l|}
\hline GFP \\
\hline 5xAGA-GFP \\
\hline 5xAGG-GFP \\
\hline 3xATA-GFP \\
\hline 5xCCC-GFP \\
\hline 5xCGG-GFP \\
\hline 5xCTA-GFP \\
\hline 3xGGA-GFP \\
\hline
\end{tabular}


SixPack

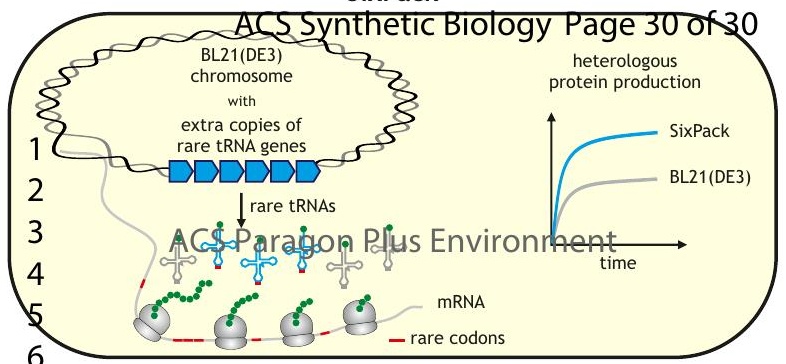

